Old Dominion University

ODU Digital Commons

2020

\title{
Numerical Modeling of Submicron Particles for Acoustic Concentration in Gaseous Flow
}

Jizhou Liu

Xiaodong Li

Fang Q. Hu

Old Dominion University, fhu@odu.edu

Follow this and additional works at: https://digitalcommons.odu.edu/mathstat_fac_pubs

Part of the Biotechnology Commons, and the Mathematics Commons

\section{Original Publication Citation}

Jizhou, L., Xiaodong, L., \& Hu, F. Q. (2020). Numerical modeling of submicron particles for acoustic concentration in gaseous flow. Journal of the Acoustical Society of America, 147(1), 152-160. doi:10.1121/10.0000568

This Article is brought to you for free and open access by the Mathematics \& Statistics at ODU Digital Commons. It has been accepted for inclusion in Mathematics \& Statistics Faculty Publications by an authorized administrator of ODU Digital Commons. For more information, please contact digitalcommons@odu.edu. 


\title{
Numerical modeling of submicron particles for acoustic concentration in gaseous flow
}

\author{
Jizhou Liu, ${ }^{1}$ Xiaodong $\mathrm{Li}^{1}{ }^{1, \mathrm{a})}$ and Fang Q. $\mathrm{Hu}^{2}$ \\ ${ }^{1}$ School of Energy and Power Engineering, Beihang University, Beijing, 100191, China \\ ${ }^{2}$ Department of Mathematics and Statistics, Old Dominion University, Norfolk, Virginia 23508, USA
}

\begin{abstract}
:
This paper intends to explore the rationality and feasibility of modeling dispersed submicron particles in air by a kinetic-based method called the unified gas-kinetic scheme (UGKS) and apply it to the simulation of particle concentration under a transverse standing wave. A gas-particle coupling scheme is proposed where the gas phase is modeled by the two-dimensional linearized Euler equations (LEE) and, through the analogous behavior between the rarefied gas molecules and the air-suspended particles, a modified UGKS is adopted to estimate the particle dynamics. The Stokes' drag force and the acoustic radiation force applied on particles are accounted for by introducing a velocitydependent acceleration term in the UGKS formulation. To validate this methodology, the computed concentration patterns are compared with experimental results in the literature. The comparison shows that the adopted LEEUGKS coupling scheme could well capture the concentration pattern of suspended submicron particles in a channel. In addition, numerical simulations with varying standing wave amplitudes, different acoustic radiation force to drag force ratios, and mean flow velocities are conducted. Their respective influences on the particle concentration pattern and efficiency are analyzed. (C) 2020 Acoustical Society of America. https://doi.org/10.1121/10.0000568
\end{abstract}

(Received 19 June 2019; revised 13 December 2019; accepted 16 December 2019; published online 21 January 2020)

[Editor: Steffen Marburg]

Pages: 152-160

\section{INTRODUCTION}

Particle and cell manipulation with acoustofluidics techniques has become a promising inter-discipline methodology drawing attention from the fields of fluid mechanics, acoustics, and bio-technology. Due to its ability to effectively and non-invasively control very small particles in fluid, it has been successfully applied for analysis purposes on many micro-sized systems like micro-electro-mechanical systems and lab-on-a-chip devices. ${ }^{1-3}$ By the use of standing wave fields, ${ }^{4-6}$ dispersed objects could be efficiently organized into certain patterns regardless of their shape, size, and polarity. With special designs of the flow field, specific concentration, separation, and size-dependent sorting of particles could also be realized.

For liquid-based systems, the above-mentioned phenomena could be well observed in laboratory experiments, such as the effect of positive and negative contrast factors on particle movement direction ${ }^{7}$ and the particle size fractionation by acoustic radiation force. ${ }^{8,9}$ In the meantime, investigations for gas-suspended particles were also carried out in channel flow configurations. ${ }^{10-12}$ Due to the low acoustic frequency, particle concentration patterns deviate slightly from the acoustic radiation force theory. ${ }^{13,14}$

While experimental observations have shown the promise of acoustofluidics to concentrate or separate particles from the medium, numerical methods were also developed to simulate the scenario of particle manipulation

\footnotetext{
${ }^{a)}$ Electronic mail: lixd@buaa.edu.cn, ORCID: 0000-0001-8685-9139.
}

under acoustic loadings. Buyukkocak et al. ${ }^{15}$ applied a Monte Carlo type method in simulating trajectories of 750 particles in a standing wave field. Fractionation of particles with different sizes and densities were simulated, and comparison with experimental measurements showed good agreement. Due to a flexibility in choosing property parameters, numerical simulations make it easy to compare particle behavior of arbitrary size and to study the relative importance of the acoustic radiation force and the acoustic streaming force (drag force). Muller et al. ${ }^{16}$ established a numerical process based on the particle tracing method in COMSOL software that revealed the particle motion transitions from being dominated by streaming-induced drag to being dominated by radiation forces as a function of particle size, channel geometry, and material properties.

When modeling the particle phase numerically, most existing simulation studies adopt the Lagrangian description in which equation of motion for each particle is solved. This idea is intrinsic for particle simulation ${ }^{17}$ but encounters computational difficulty for small diameter and high number density cases. For particle diameter less than $1 \mu \mathrm{m}$ its number density practically exceeds $10^{12} \mathrm{~m}^{-3}$, which makes the complete simulation of all particles very time-consuming. However, one advantage of the Lagrangian description is that it could consider the particle collision and aggregation process, yet the aggregation effect for submicron particles is less obvious even at high sound pressure level (SPL). ${ }^{18}$

When particles are of a small size and high density, they would have a dynamic behavior that sits between the continuum gas medium and the complete discrete particulate objects 
by analogy. Therefore, for submicron range particles, the adoption of kinetic methods which were developed for treating rarefied gas dynamics would be appropriate. However, in addition to rarefied gas dynamics where particle transport and collision processes are dominant, suspended particles in acoustic field also undergo viscous drag force and acoustic radiation force from the medium. These two external forces introduce an acceleration term in the governing equations of particle phase. Passalacqua and Fox ${ }^{19}$ modeled the particle phase by the Boltzmann equation with viscous acceleration term. They built a continuum-kinetic coupling model for gas-particle flow and solved the kinetic equation of the particle phase with a third order quadrature-based method of moment (QMOM). With the same modeling assumption, Liu et al. ${ }^{20}$ solved the particle dynamics with the unified gas-kinetic scheme (UGKS). By coupling the UGKS with the gas-kinetic scheme (GKS), Liu's model for gas-particle two-phase flow showed multiscale property in capturing the particle trajectory crossing (PTC), the particle wall reflecting phenomena and the vortexinduced segregation of inertial particles under different Stokes numbers.

As is shown in these studies, kinetic modeling for the particle phase provides a potential treatment for gas-particle two-phase flow simulations. Based on the Boltzmann equation, the UGKS integrates the collision and the free transport of gas molecules which makes it valid for a varying range of flow regime from continuum to rarefied. ${ }^{21,22} \mathrm{By}$ consequence, the present work intends to combine this modeling idea with the UGKS and apply it to the simulation of particle concentration phenomenon by acoustic loadings. Specifically, a gas-particle coupling scheme is built with the linearized Euler equations for the gas phase and the modified UGKS for modeling the particle dynamics. The coupling in the proposed scheme considers momentum exchange where the particle phase undergoes forces from the gas phase. The established scheme should be able to predict some of the observations in the literature and provide an efficient alternative to the Lagrangian modeling especially for nano-sized and sub micro-sized particles. To validate the proposed numerical modeling, submicron particle concentration by transverse standing wave in a two-dimensional (2-D) micro channel is simulated. The simulated results are first compared with experimental measurements. ${ }^{11}$ Then parametric studies on the SPL of standing wave, the acoustic radiation force (ARF) to drag force (DF) ratio, and the mean flow velocity are conducted.

In Sec. II the modified UGKS with external force term for modeling dispersed submicron particles is described. Treatment of the acceleration term and algorithm to speed up the calculation are presented in detail. Section III outlines the formulation of the problem and the corresponding linearized Euler equations (LEE)-UGKS coupling scheme. In Sec. IV, the proposed scheme is employed to study the particle acoustic concentration phenomenon by comparing with experimental observations in the literature and by parametric studies.

\section{MODIFIED UGKS FOR SUBMICRON PARTICLE PHASE}

\section{A. UGKS with external forces}

The UGKS is by origin a kinetic method. The same as in many other kinetic formulations, the particle state is described by a density distribution function in time, spatial, and velocity space $f\left(t, \mathbf{x}, \mathbf{u}_{\mathbf{p}}\right)$. Suppose that the dispersed particles are mono-disperse the governing equation with external force term is ${ }^{20}$

$$
\frac{\partial f}{\partial t}+\nabla_{\mathbf{x}}\left(\mathbf{u}_{p} f\right)+\nabla_{\mathbf{u}}(\mathbf{A} f)=\mathbb{C},
$$

where

$$
\begin{aligned}
& \mathbf{A}=\frac{1}{\tau_{s t}}\left(\mathbf{U}_{g}-\mathbf{u}_{p}\right)+\frac{F_{A R F}}{m_{p}}, \quad \tau_{s t}=\frac{4 \rho_{m p} d_{p}^{2}}{9 \mu_{g}} C(K n), \\
& \mathbb{C}=\frac{g-f}{\tau}, \tau=\frac{4 \sqrt{\pi}}{3} n_{p} \sqrt{\frac{2 k_{B} T_{p}}{m_{p}}} d_{p}^{2} .
\end{aligned}
$$

Subscript $p$ denotes properties and variables related with the particle phase and subscript $g$ denotes those of the gas phase. In Eq. (1), $\mathbf{A}$ is the acceleration term brought about by the external forces on the particle phase. Within the scope of this work, the external forces are the viscous drag force and the acoustic radiation force. $\tau_{s t}$ denotes the relaxation time of the viscous drag effect, it is related to the properties of particles and the medium. $F_{\mathrm{ARF}}$ represents the acoustic radiation force, which is not velocity dependent. $m_{p}, \rho_{m p}$, and $d_{p}$ are the mass, material density, and diameter of particle. $\mu_{g}$ is the dynamic viscosity of gas. As the Reynolds number of the flow around particle is supposed to be less than unity in this study, the Stokes' drag force law with Cunningham correction, ${ }^{23} C(K n)$, is employed here. $K n$ is the Knudsen number of the flow. The term $\mathbb{C}$ is the Bhatnagar-Gross-Krook (BGK) collision operator where $g$ is the particle distribution function in equilibrium state and $\tau$ is the relaxation time. In the expression of $\tau, n_{p}$ and $T_{p}$ denote the number density and the temperature of the particle phase. $k_{B}$ is the Boltzmann constant.

Without the acceleration term, the conventional UGKS adopts a finite volume formulation for the spatial coordinates and a discrete velocity space. Take a one-dimensional case as an example. The spatial domain is discretized into $I$ control volumes and the velocity space is discretized by $K$ points. For the $i$ th control volume at the $k$ th discrete velocity, the update of cell average distribution function is

$$
\begin{aligned}
f_{i, k}^{n+1}= & f_{i, k}^{n}+\frac{1}{\Delta x} \int_{t^{n}}^{t^{n+1}} u_{k}\left(F_{i-1 / 2, k}-F_{i+1 / 2, k}\right) d t \\
& +\frac{\Delta t}{2}\left(\frac{g_{i, k}^{n+1}-f_{i, k}^{n+1}}{\tau^{n+1}}+\frac{g_{i, k}^{n}-f_{i, k}^{n}}{\tau^{n}}\right) .
\end{aligned}
$$

The distribution function $F_{i+1 / 2, k}$ at cell interface has a specific construction process that contains particle free 
transport and collision mechanisms, which is given in detail in Ref. 24.

However, the velocity gradient in the acceleration term in Eq. (1) could not be dealt with by the conventional UGKS formulation. To treat this problem, Liu et al. ${ }^{20}$ applied a finite volume method for both the spatial and velocity coordinates. While their formulation is complete, it is tedious to understand every aspect in it. In this study, we seek a simplified treatment of the acceleration term that could guarantee a correct momentum exchange between the two phases. In detail, we stick to a finite difference formulation for the velocity space. By moving the acceleration term to the right hand side of Eq. (1), an equivalent form is obtained,

$$
\frac{\partial f}{\partial t}+\nabla_{\mathbf{x}}\left(\mathbf{u}_{p} f\right)=\tilde{\mathbb{C}},
$$

where

$$
\tilde{\mathbb{C}}=\frac{g-f}{\tau}-\frac{\left(\mathbf{U}_{\mathbf{g}}-\mathbf{u}_{p}\right) \frac{\partial f}{\partial \mathbf{u}_{\mathbf{p}}}}{\tau_{s t}}-\frac{F_{A R F}}{m_{p}} \frac{\partial f}{\partial \mathbf{u}_{\mathbf{p}}} .
$$

This treatment converts the effect of external force acceleration to be part of the collision effect except that this "collision" happens between particles and surrounding medium. For Eq. (3), the conventional UGKS formulation could be directly applied to form Eq. (2). The thing that is changed is the newly derived collision term $\tilde{\mathbb{C}}$. In this new term, the only unknown is the first order derivative of the distribution function but it could be calculated by the finite difference method in the velocity space. ${ }^{25}$

\section{B. Adaptive velocity interval algorithm}

In the UGKS, the density distribution function in equilibrium state is assumed to be of Maxwellian type. For a 2$\mathrm{D}$ case, it has the form of a normal distribution:

$$
g(t, x, y, u, v)=\rho_{p}(t, x, y)\left(\frac{\lambda}{\pi}\right)^{3 / 2} e^{-\lambda\left(\left(u_{p}-U_{p}\right)^{2}+\left(v_{p}-V_{p}\right)^{2}+\xi^{2}\right)} .
$$

The characteristic velocity $u_{c}=\lambda^{-1 / 2}=\sqrt{2 k_{B} T / m_{p}}$ determines the half width of the normal distribution function. For example, in gas dynamics problems, the characteristic velocity usually stands for the sound speed. However, when the submicron particle is modeled, its molecular mass is about $10^{9}$ times heavier than that of a gas molecule, which results in a very small characteristic velocity $\left(u_{c} \approx 6 \times 10^{-3} \mathrm{~m} / \mathrm{s}\right)$. Besides, the motion of the dispersed submicron particles is coupled with the gas phase. For a SPL around $150 \mathrm{~dB}$, the maximum gas velocity is about $1.58 \mathrm{~m} / \mathrm{s}$. These two facts together mean that the density distribution function is very "narrow" in the velocity space (determined by the characteristic velocity), but has a very large moving interval (coupled with gas motion) as is shown in Fig. 1.

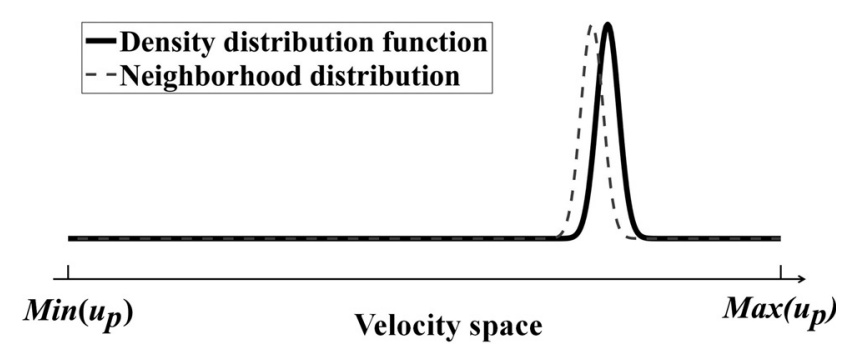

FIG. 1. Typical density distribution function and the potential moving interval in the velocity space when modeling submicron particles with the UGKS.

This special feature shown in Fig. 1 could make many kinetic-based methods to be computationally inefficient since non-zero information exists only in a very limited velocity range. However, if the velocity field of the gas phase is without shock, the density distribution functions in adjacent control volumes should share a certain range of velocity interval as is shown by the solid black line and the dotted gray line in Fig. 1. Since the UGKS employs second order spatial scheme, only the nearest neighbors are involved in the interpolation process.

With the above considerations, an adaptive velocity interval algorithm is applied herein in the interpolation and update processes to reduce the amount of calculations. For the $i$ th control volume at time step $t^{n}$, its density distribution function has a compact support in the velocity interval $\left[U_{c}^{i}-R^{i}, U_{c}^{i}+R^{i}\right]$ with $U_{c}^{i}$ the expectation velocity and $R^{i}$ the supporting radius. In the spatial interpolation of the density distribution function, only cell $i-1$ and $i+1$ are involved. Therefore all useful information will be confined in the effective interval $\left[\min \left(U_{c}^{i-1}-R^{i-1}, U_{c}^{i}-R^{i}, U_{c}^{i+1}\right.\right.$ $\left.\left.-R^{i+1}\right), \max \left(U_{c}^{i-1}+R^{i-1}, U_{c}^{i}+R^{i}, U_{c}^{i+1}+R^{i+1}\right)\right]$. By restricting the calculation within this effective velocity interval, the total workload is obviously reduced. For time step $t^{n+1}$, since the particle mean velocity is updated, $U_{c}^{i}$ is also updated according to the new density distribution function. This makes the algorithm adaptive. We also note that this does not mean that only the distribution function in the effective velocity interval needs to be stored. In practice, distribution function in the entire discrete velocity range is stored.

\section{PROBLEM STATEMENT AND THE GAS-PARTICLE COUPLING SCHEME}

To validate the kinetic modeling of submicron particles, the modified UGKS is coupled with the linearized Euler equations to formulate a continuum-kinetic coupling scheme for gas-particle two-phase flows. Here the problem simulated concerns the particle concentration phenomenon under transverse standing wave in a micro channel.

\section{A. Problem description and assumptions}

Figure 2 sketches the problem geometry as in the experimental investigation by Imani and Robert. ${ }^{11}$ The particle-seeded air stream is introduced from the inflow 


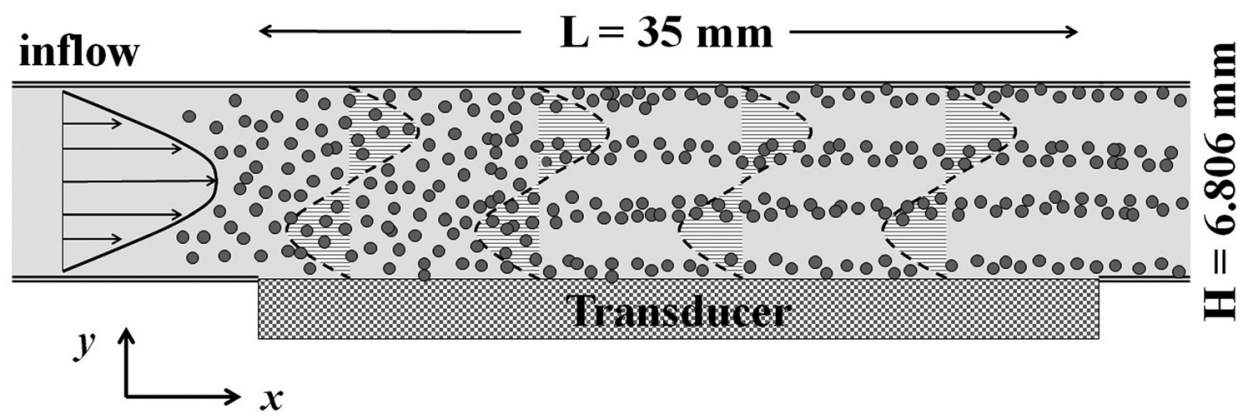

FIG. 2. Schematic representation of the experimental setup.

side. An electrostatic transducer of $L=35 \mathrm{~mm}$ in length is mounted into the channel wall. It is able to generate a maximum SPL of $154.5 \mathrm{~dB}$. To guarantee a complete wavelength in the transversal direction, the working frequency is set to be $50 \mathrm{kHz}$ which corresponds to a channel height of $H=6.806 \mathrm{~mm}$. At the outlet, an optic system is installed to measure the particle density and size. The seeded particle is solid $\mathrm{TiO}_{2}$ of $300 \mathrm{~nm}$ in diameter initially. Its material density is $3900-4200 \mathrm{~kg} / \mathrm{m}^{3}$. At the outlet, the average particle size grows to $460 \mathrm{~nm}$ as is caused by the acoustic aggregation effect.

From experiment to numerical simulation, the following assumptions are made.

(1) Particles do not cohere: as is shown in the experimental study, the aggregation effect is not very important for submicron particles. With this assumption, particle modeling with the UGKS is possible because the Boltzmann equation is only able to deal with monodisperse particle.

(2) Sinusoidal and continuous standing wave form: assume that the standing wave pressure field has a continuous cosine form even though SPL could reach $150 \mathrm{~dB}$. Under this condition, the acoustic radiation force has an analytical expression.

(3) Laminar gas mean flow along the channel: since the Reynolds number is relatively small $(R e \leq 100)$, the mean flow field is considered laminar. The velocity profile is calculated based on the Poiseuille condition.

(4) Displacement of the transducer is neglected: fixed wall condition is assumed on both sides of the channel, the transverse standing wave is given by imposing appropriate initial conditions.

\section{B. Gas-particle coupling scheme}

With the above assumptions, the configuration in Fig. 2 could be simplified to a two-dimensional channel flow with particles and transverse standing wave. In this simplified configuration, there are two essential mechanisms of particle motions: particle convection along the channel and particle concentration by the standing wave in the transversal direction.

To maintain the above-mentioned mechanisms, a LEEUGKS coupling scheme for the gas-particle flow is proposed. The linearized Euler equations ${ }^{26}$ are employed because it could describe the transverse standing wave as well as the mean flow motion along the $x$ axis of the channel for the gas phase. The governing equations are as follows:

$$
\frac{\partial \mathbf{Q}_{g}}{\partial t}+\mathbf{A} \frac{\partial \mathbf{Q}_{g}}{\partial x}+\mathbf{B} \frac{\partial \mathbf{Q}_{g}}{\partial y}+\mathbf{C} \mathbf{Q}_{g}=\mathbf{S},
$$

in which

$$
\begin{aligned}
& \mathbf{Q}_{g}=\left(\begin{array}{c}
\rho_{g} \\
u_{g} \\
v_{g} \\
p_{g}
\end{array}\right) \mathbf{A}=\left(\begin{array}{cccc}
\bar{U}_{g} & \bar{\rho}_{g} & 0 & 0 \\
0 & \bar{U}_{g} & 0 & \frac{1}{\bar{\rho}_{g}} \\
0 & 0 & \bar{U}_{g} & 0 \\
0 & 1 & 0 & \bar{U}_{g}
\end{array}\right) \quad \mathbf{B}=\left(\begin{array}{cccc}
0 & 0 & \bar{\rho}_{g} & 0 \\
0 & 0 & 0 & 0 \\
0 & 0 & 0 & \frac{1}{\bar{\rho}_{g}} \\
0 & 0 & 1 & 0
\end{array}\right) \quad \mathbf{C}=\left(\begin{array}{cccc}
0 & 0 & \frac{d \bar{\rho}_{g}}{d y} & 0 \\
0 & 0 & \frac{d \bar{U}_{g}}{d y} & 0 \\
0 & 0 & 0 & 0 \\
0 & 0 & 0 & 0
\end{array}\right), \\
& \mathbf{S}=\left(\begin{array}{ll}
0-\frac{\rho_{p}}{\tau_{s t}}\left(\bar{U}_{g}+u_{g}-u_{p}\right) & -\frac{\rho_{p}}{\tau_{s t}}\left(\bar{V}_{g}+v_{g}-v_{p}\right) \quad-\frac{\rho_{p}}{\tau_{s t}}\left(u_{p}\left(\bar{U}_{g}+u_{g}-u_{p}\right)+v_{p}\left(\bar{V}_{g}+v_{g}-v_{p}\right)\right)
\end{array}\right) .
\end{aligned}
$$

Variables with over bar stand for mean flow quantities.

On the other hand, the particle phase is modeled by the modified UGKS with external force Eq. (1). Here we only focus on the momentum exchange between the two phases and assume that the temperature of particles is the same as the local temperature of the gas phase. Due to a low volume 
fraction of particle phase, $\rho_{p} / \rho_{g}$ is practically a small number (less than 0.01).

\section{Computational methods and setups}

The simulation of the acoustic concentration effect usually covers several thousand wave periods in time. To have a minimum wave distortion in long term simulations, computational aeroacoustics methods are applied to solve the gas phase equations. The spatial interpolation is based on the fourth order dispersion-relation-preserving (DRP) finite difference scheme. ${ }^{27}$ The time stepping employs the optimized four-level Adams-Bashforth method. ${ }^{25}$ As for the wall boundaries, a ghost-point based high-order wall condition is used.

For the submicron particle phase, the modified UGKS with adaptive velocity interval algorithm is adopted as is detailed in Sec. II B. Due to the derivative term in Eq. (3), the trapezoidal rule in Eq. (2) could no longer be employed for the BGK-type collision term. Here we return to the explicit Euler forward method. To enhance the computing speed, MPI-based parallel code is developed for this 2-D LEE-UGKS coupling scheme.

The main parameters and conditions for the simulations are listed in Table I.

The computational domain is a rectangle of $L \times H$ as is shown in Fig. 2. It is divided into $N_{x} \times N_{y}=120 \times 48$ control volumes. Within each control volume, the velocity space is further discretized under the framework of the UGKS. Depending on the mean flow speed $U_{\text {mean }}$, the discretization in the velocity space takes $N_{u} \times N_{v}=49 \times 169$ (for cases with $U_{\text {mean }}=0$ and $0.05 \mathrm{~m} / \mathrm{s}$ ) and $N_{u} \times N_{v}$ $=89 \times 177\left(U_{\text {mean }}=0.1 \mathrm{~m} / \mathrm{s}\right)$, respectively. Since the adaptive velocity interval algorithm is used, the actual amount of degrees of freedom in real-time calculation is much smaller than these indicated values. The time step is $\Delta t=2.5 \times 10^{-8}$ second which corresponds to $1 / 800$ of the standing wave period. The simulations were run on a cluster

TABLE I. Main parameters and conditions for the gas phase and the particle phase.

\section{Gas phase}

Density, pressure and temperature

Standing wave frequency $f_{g}$

Channel mean flow speed $U_{\text {mean }}$

Channel velocity profile $\bar{U}_{g}(y)$

Standing wave pressure level

Initial conditions
Standard atmospheric condition $50 \mathrm{kHz}$

$0 \mathrm{~m} / \mathrm{s}, 0.05 \mathrm{~m} / \mathrm{s}, 0.1 \mathrm{~m} / \mathrm{s}$

$\bar{U}_{g}(y)=\frac{\left(6 U_{\text {mean }}\right)}{H^{2}}(H-y) y$

$140-150 \mathrm{~dB}$

$\rho_{g}=\rho_{0} \cos (2 \pi y / H)$

$u_{g}=0 ; v_{g}=0$

$p_{g}=p_{0} \cos (2 \pi y / H)$

Particle phase

Material density $\rho_{m p}$

Particle diameter $d_{p}$

Number density $n_{p}$

Initial conditions

\section{$4000 \mathrm{~kg} / \mathrm{m}^{3}$ $460 \mathrm{~nm}$ $1 \times 10^{12} \mathrm{~m}^{-3}$ \\ $\rho_{p}=m_{p} n_{p}$ \\ $u_{p}=\bar{U}_{g} ; v_{p}=0$}

with Intel E5-2683 CPU devices. For the $U_{\text {mean }}=0.05 \mathrm{~m} / \mathrm{s}$ test case, the computation speed was 2.086 timesteps/s with 30 MPI-connected CPU threads.

For the plane standing wave in y-direction, the acoustic radiation force derived by Gor'kov ${ }^{14}$ is

$$
F_{A R F}=C \frac{\pi k d_{p}^{3}}{2} E_{a c} \Phi \sin (2 k y),
$$

where

$$
E_{a c}=\frac{1}{4} \kappa_{0} p_{a}^{2}, \quad \Phi=\frac{\rho_{p}+2 / 3\left(\rho_{p}-\rho_{g}\right)}{2 \rho_{p}-\rho_{g}}-\frac{\beta_{p}}{\beta_{g}},
$$

$E_{a c}$ is the acoustic energy density related with the acoustic pressure and $\Phi$ is the so-called acoustic contrast factor related with the compressibility of the gas phase and the particle phase. With a $\sin (2 k y)$ spatial modulation, the acoustic radiation force pushes particles toward pressure nodes at $0.25 \mathrm{H}$ and $0.75 \mathrm{H}$. However, for the current simulation, the acoustic radiation force is less dominant because the particle diameter is small and the working frequency is low. Therefore the effect of viscous drag force should be considered as well.

In this work, both the gas and particle phases are solved in time domain. However, the theoretical expression for the acoustic radiation force in Eq. (5) is time-averaged. To deal with this inconsistency, we assume that the acoustic radiation force oscillates periodically in time domain with the same frequency as the standing wave because it is the standing wave that generates the acoustic radiation force. Therefore we multiply $\sin \left(2 \pi f_{g} t\right)$ to the right hand side of Eq. (6) in the simulations.

\section{SIMULATION RESULTS AND DISCUSSIONS}

With the numerical scheme and the parameter settings described in Sec. III, a series of simulations are conducted with different standing wave SPLs, acoustic radiation force to drag force ratios and mean flow velocities to study their relative influences on particle concentration efficiency and pattern.

\section{A. Comparison with experimental measurements}

To validate the numerical modeling, the simulated particle density profile with the proposed LEE-UGKS coupling scheme is first compared with experimental measurements in Ref. 11. The amplitude of standing wave is set to be $149.63 \mathrm{~dB}_{\text {rms. }}$. The ARF/DF ratio is on average $76.83 \%$. The simulation is done without mean flow for 7500 periods of standing wave. As could be interpreted from Fig. 3, the LEE-UGKS scheme is able to get a similar particle concentration pattern as in the experimental study. Two enriched peaks form near the centerline of the channel with a peak value of approximately 2 . The separation of the two peaks is about $0.15 \mathrm{H}$ which is very close to the experimental measurement. The numerical result is completely symmetric 


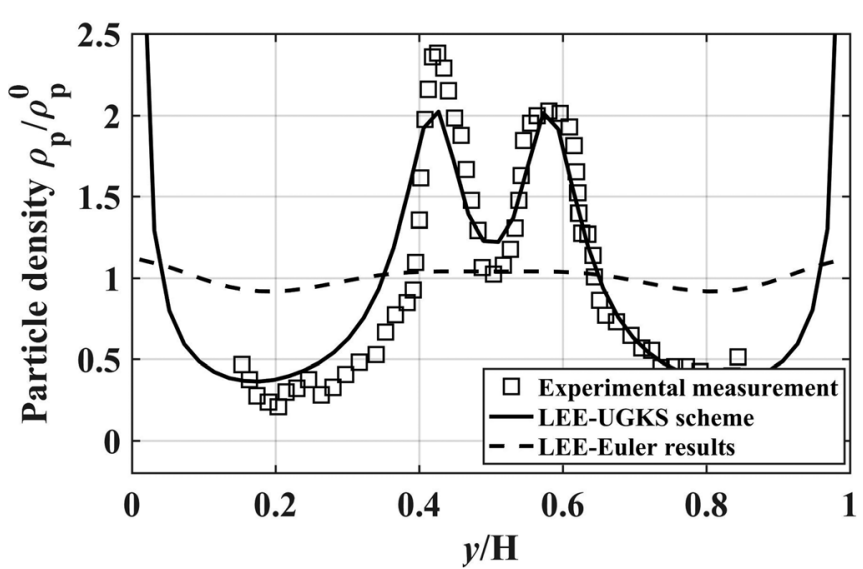

FIG. 3. Particle concentration comparison between experimental measurement in Ref. 11, kinetic modeling method (LEE-UGKS scheme) and continuum modeling (LEE-Euler method). $\rho_{p}$ is the post-concentration particle density and $\rho_{p}^{0}$ denotes the initial particle density.

because we assume the same wall condition on both sides and continuous wave form. While in the experiment, the displacement of the transducer will cause a certain level of asymmetry as could be noticed in Fig. 3. Owing to the mass conservation, particles also concentrate near the side walls.

As a reference, the dashed line in the figure shows the particle density calculated using a two-fluid model (TFM). In this method the particle phase is considered continuum and is solved by the Euler equations. Compared with the experimental measurement, the two-fluid model could not predict satisfactory peak values as the LEE-UGKS scheme does. The observed difference between the two modeling methods might lie in their fundamental assumptions, respectively. For the continuum modeling, all particles inside one control volume have only one unique velocity so that they move at the same pace. However, in the kinetic modeling, a distribution function $f\left(t, \mathbf{x}, \mathbf{u}_{\mathbf{p}}\right)$ is employed to describe particles' states. This means that particles within the same control volume could have different velocities. For example, the second term of the collision term $\tilde{\mathbb{C}}$ in Eq. (3) denotes the momentum exchange rate between the gas phase and the particle phase due to the viscous drag force. Since the distribution function $f\left(t, \mathbf{x}, \mathbf{u}_{\mathbf{p}}\right)$ is velocity-dependent, particles with different velocities would gain or lose different amount of momentum even if they belong to the same control volume, which is closer to the reality.

\section{B. Influence of SPL on concentration efficiency}

By varying the amplitude of the standing wave, the influence of SPL on particle concentration efficiency is studied with a fixed ARF/DF ratio. The SPL ranges from 143.18 to $149.63 \mathrm{~dB}_{\mathrm{rms}}$. The total simulation time is also set to 7500 periods. The concentration efficiency $\eta$ is defined as the relative increase of particle density in the enriched bands region, ${ }^{11}$

$$
\eta=\frac{\int_{y_{l}}^{y_{r}}\left(\rho_{c o n}-\rho_{i n i}\right) d y}{\rho_{\text {ini }}\left(y_{r}-y_{l}\right)} .
$$

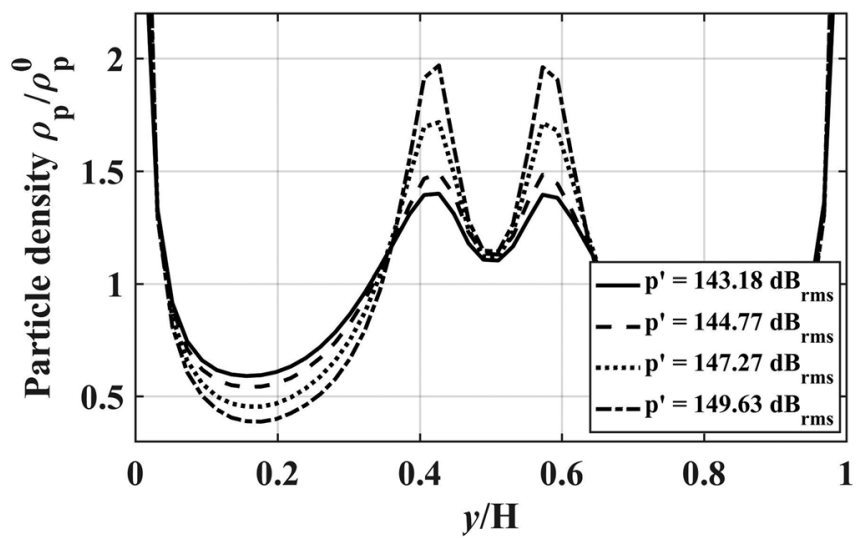

FIG. 4. Particle concentration patterns at different SPLs with a fixed ARF/ $(\mathrm{ARF}+\mathrm{Drag})$ ratio of $0.4345 . \rho_{p}$ is the post-concentration particle density and $\rho_{p}^{0}$ denotes the initial particle density.

In Eq. (6), $\rho_{\text {ini }}$ and $\rho_{\text {con }}$ denote the initial and postconcentration particle density. $y_{l}$ and $y_{r}$ are left and right bounds in y direction for the concentrated peaks. The simulated particle density profiles are shown in Fig. 4. It could be seen that increasing pressure amplitude of the standing wave will increase the particle density in the enriched bands. The concentration efficiency depends nearly linear on the SPL in decibels as shown in Fig. 5.

\section{ARF/DF ratio on concentration pattern}

While the SPL of the standing wave determines particle concentration efficiency, the relative importance of the acoustic radiation force and the drag force affects the particle concentration pattern. For the current parameter settings, the acoustic radiation force is no longer dominant due to small particle size and low working frequency. Imani and Robert $^{28}$ estimated that acoustic radiation force represented approximately $40 \%$ of total force for particles with diameters of $200 \mathrm{~nm}$.

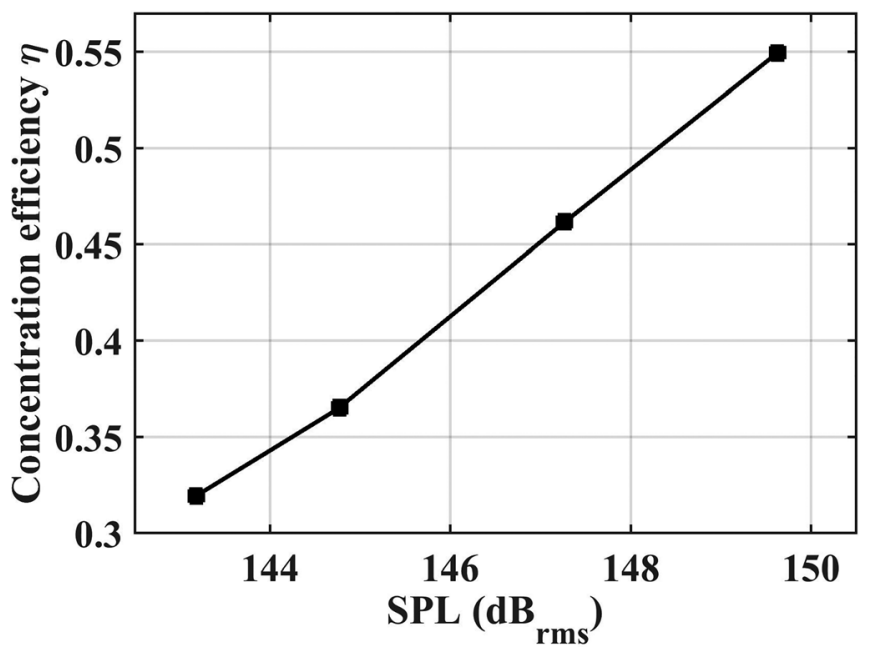

FIG. 5. Particle concentration efficiency as a function of SPL. 


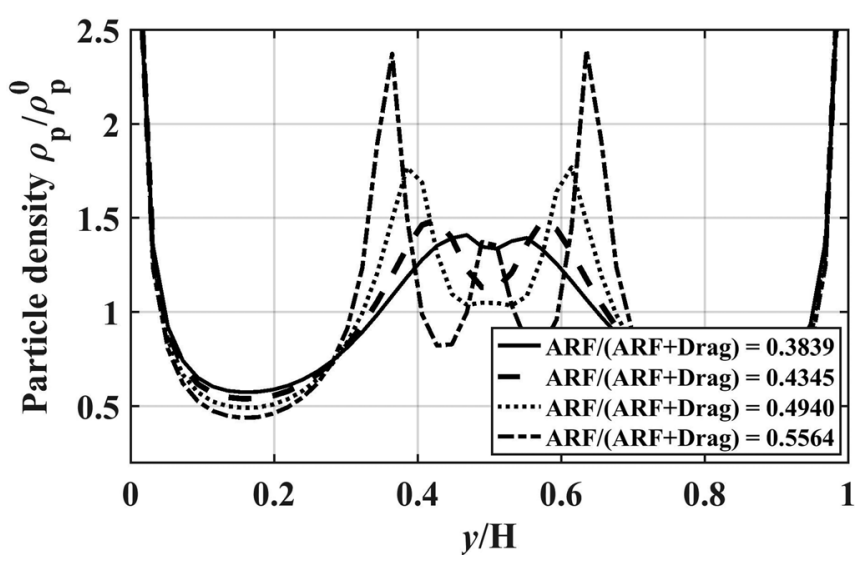

FIG. 6. Particle concentration patterns of different $\mathrm{ARF} /(\mathrm{ARF}+\mathrm{DF})$ ratios. $\rho_{p}$ is the post-concentration particle density and $\rho_{p}^{0}$ denotes the initial particle density.

In our numerical investigations we could not determine the exact value of the acoustic radiation force but the ARF/ DF ratio could be altered artificially to show how this ratio influences the concentration pattern. With a fixed SPL at $144.77 \mathrm{~dB}_{\mathrm{rms}}$ and simulation time of 7500 periods, ARF/ $(\mathrm{ARF}+\mathrm{DF})$ ratios ranging from 0.38 to 0.55 are simulated. The force ratio is estimated by period-averaged value. From Fig. 6 it could be concluded that stronger acoustic radiation force would lead to enriched bands to have higher peak values and larger separation distances. This trend is consistent to the known effect on the acoustic radiation force since it tends to bring particles to the pressure nodes. Two extreme cases where either the ARF or the drag force is dominant are also tested. In Fig. 7, when there is only drag force that acts on the particles, particles group around the centerline of the channel in one peak. ${ }^{28}$ By contrast, if the acoustic

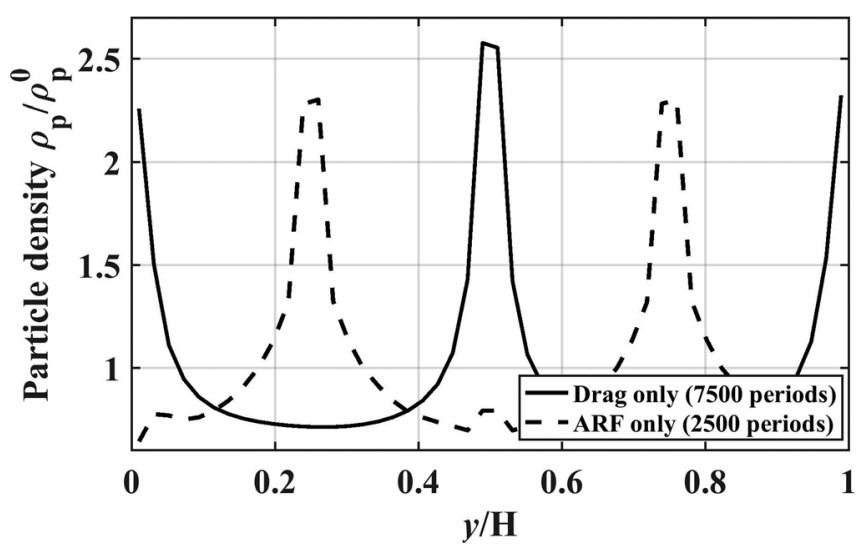

FIG. 7. Particle concentration patterns of ARF only and drag force only cases. $\rho_{p}$ is the post-concentration particle density and $\rho_{p}^{0}$ denotes the initial particle density.

radiation force is dominant, particles are entrained towards the pressure nodes of the standing plane wave which agrees with the theoretical prediction and experimental observations in the literature.

\section{Particle concentration with mean flow velocity}

After parametric studies without mean flow shown above, the whole scenario of particle concentration by standing wave and convection along the channel is simulated with mean flow velocities of 0.05 and $0.1 \mathrm{~m} / \mathrm{s}$. The SPL is set to be $149.63 \mathrm{~dB}_{\mathrm{rms}}$ and the contours of particle density after 7500 periods are shown in Fig. 8. Darker color denotes higher particle density.

Particles are introduced with uniform density from $x=0$. As they move downstream the channel, they undergo the influence of the acoustic radiation force and the drag

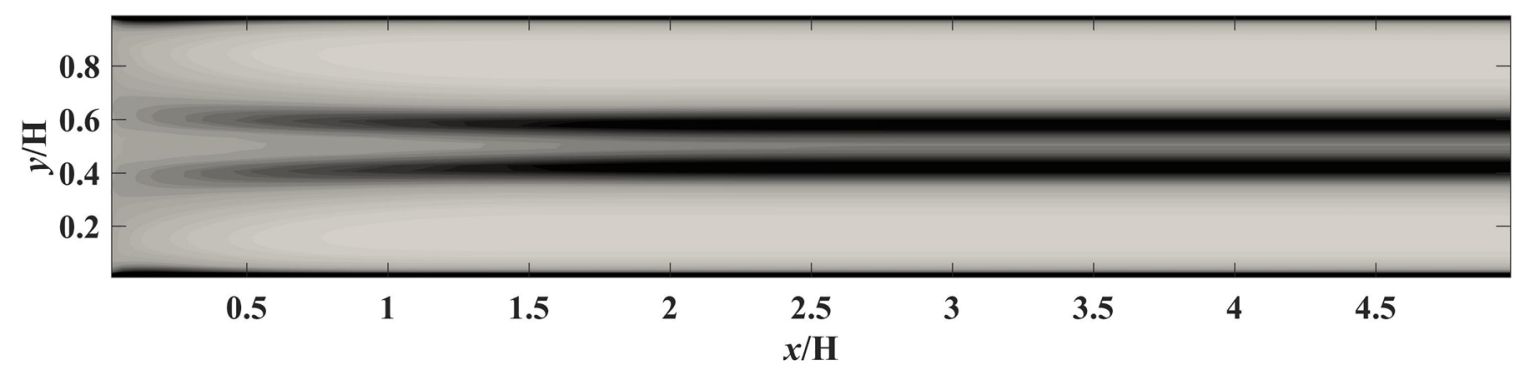

(a)

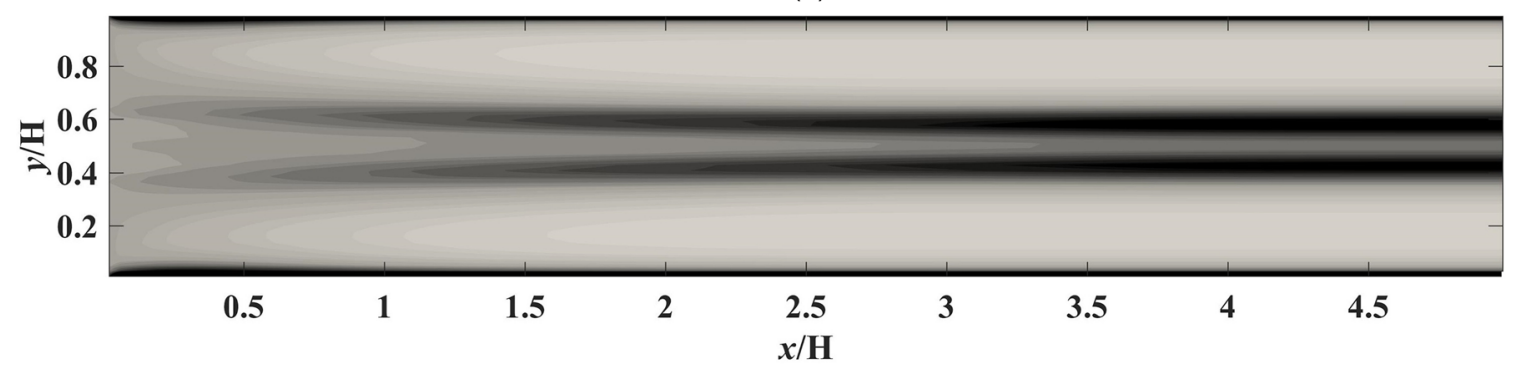

(b)

FIG. 8. (Color online) Contours of particle density in channel (a) $U_{\text {mean }}=0.05 \mathrm{~m} / \mathrm{s}$ and (b) $U_{\text {mean }}=0.1 \mathrm{~m} / \mathrm{s}$, darker shading represents regions with higher particle density. 
force that lead them to a certain concentrated pattern. By Figs. 8(a) and 8(b), it could be concluded that the mean flow velocity controls the particle concentration pattern in the channel. For the $U_{\text {mean }}=0.05 \mathrm{~m} / \mathrm{s}$ case, the axial position where particles are clearly concentrated is $x=1.5 \mathrm{H}$ and this position for the $U_{\text {mean }}=0.1 \mathrm{~m} / \mathrm{s}$ case is about $\mathrm{x}=3 \mathrm{H}$. In other words, at a given axial position, smaller mean velocity lead to higher concentration efficiency. For example, at $\mathrm{x}=2.5 \mathrm{H}$, the concentration efficiencies for the two cases are 0.5021 and 0.3285 , respectively.

\section{v. CONCLUSIONS}

Based on the analogy between the gas molecules and small dispersed particles, the UGKS is modified to represent the submicron particle phase with external force effect. By the proposed 2-D LEE-UGKS coupling scheme, the acoustic concentration phenomenon of submicron particles in a micro channel is simulated.

The simulated particle concentration pattern is first compared with experimental measurements in the literature. The good agreement of the results indicates that the kinetic modeling method for submicron particles in air is able to capture major features in the studied problem. By comparison, the continuum modeling of particles is less satisfactory for the current test case. Influences of some key parameters are investigated. The concentration efficiency is shown to depend linearly on the SPL of the standing wave. The acoustic radiation force to drag force ratio is another important factor that could alter the concentration pattern. With an increasing ratio, the enriched bands where particles gather tend to have higher peak values and larger separation distances. Finally, the influence of mean velocity amplitude on the evolution of particle density along the channel is studied and analyzed.

This study demonstrates the rationality and feasibility of modeling submicron particles in air stream with the UGKS. It also shows that the established LEE-UGKS scheme is able to predict the particle concentration phenomenon under transverse standing waves. Here, we only focus on simulating mono-disperse particles of size smaller than $1 \mu \mathrm{m}$ because the computation costs increase as particle size grows due to an even narrower density distribution function in Fig. 1. In this study, we take the relative importance of the acoustic radiation force and the drag force as a valuable issue when trying to predict particle concentration pattern but the absolute amplitude of the acoustic radiation force is still an open topic.

\section{ACKNOWLEDGMENTS}

This work was supported by grants from the National Key Research and Development Project (No. 2018YFA0703300), the National Science Foundation of China (Nos. 91752204 and 91952301). Mr. J.L. would like to acknowledge the financial support from the China Scholarship Council (CSC) for his stay as a visiting Ph.D. student at the Department of Mathematics and Statistics of the Old Dominion University, VA. The authors would also like to thank the anonymous reviewers for the helpful comments and suggestions.

${ }^{1}$ J. Friend and L. Y. Yeo, "Microscale acoustofluidics: Microfluidics driven via acoustics and ultrasonics," Rev. Mod. Phys. 83(2), 647-687 (2011).

${ }^{2}$ S. Oberti, A. Neild, R. Quach, and J. Dual, "The use of acoustic radiation forces to position particles within fluid droplets," Ultrasonics 49(1), 47-52 (2009).

${ }^{3}$ G. M. Whitesides, "The origins and the future of microfluidics," Nature 442(7101), 368-373 (2006).

${ }^{4}$ E. Robert, R. Jajarmi, J. Pettersson, and K. Engvall, "Acoustic separation of submicron particles in gases," Proc. Mtgs. Acoust. 19(1), 045020 (2013).

${ }^{5}$ L. Ren, S. Yang, P. Zhang, Z. G. Qu, Z. Mao, P. Huang, Y. Chen, M. Wu, L. Wang, P. Li, and T. J. Huang, "Standing surface acoustic wave (SSAW)-based fluorescence-activated cell sorter," Small 14(40), 1801996 (2018).

${ }^{6}$ S. Orbay, A. Ozcelik, H. Bachman, and T. J. Huang, "Acoustic actuation of in situ fabricated artificial cilia," J. Micromech. Microeng. 28(2), 025012 (2018).

${ }^{7}$ F. Petersson, L. Aberg, Sward-Nilsson, Ann-Margret, and T. Laurell, "Free flow acoustophoresis: Microfluidic-based mode of particle and cell separation," Anal. Chem. 79(14), 5117-5123 (2007).

${ }^{8}$ M. Kumar, D. L. Feke, and J. M. Belovich, "Fractionation of cell mixtures using acoustic and laminar flow fields," Biotechnol. Bioeng. 89(2), 129-137 (2005).

${ }^{9}$ M. Wu, Z. Mao, K. Chen, H. Bachman, Y. Chen, J. Rufo, L. Ren, P. Li, L. Wang, and T. J. Huang, "Acoustic separation of nanoparticles in continuous flow," Adv. Funct. Mater. 27(14), 1606039 (2017).

${ }^{10}$ M. J. Anderson, R. Budwig, K. S. Line, and J. G. Frankel, "Use of acoustic radiation pressure to concentrate small particles in an air flow," in Proceedings of the IEEE Ultrasonics Symposium, Munich, Germany(October 8-11 2002), pp. 481-484.

${ }^{11}$ R. J. Imani and E. Robert, "Acoustic separation of submicron solid particles in air," Ultrasonics 63(1), 135-140 (2015).

${ }^{12}$ R. Budwig, M. J. Anderson, G. Putnam, and C. Manning, "Ultrasonic particle size fractionation in a moving air stream," Ultrasonics 50(1), 26-31 (2010).

${ }^{13}$ V. King, "On the acoustic radiation pressure on spheres," Proc. R. Soc. A 147, 212-240 (1934).

${ }^{14} \mathrm{P}$. Gor'kov, "On the forces acting on a small particle in an acoustical field in an ideal fluid," Sov. Phys. Dokl. 6(1), 773-775 (1962).

${ }^{15}$ S. Buyukkocak, M. B. Ozer, and B. Cetin, "Numerical modeling of ultrasonic particle manipulation for microfluidic applications," Microfluid. Nanofluid. 17(6), 1025-1037 (2014).

${ }^{16} \mathrm{P}$. B. Muller, R. Barnkob, M. J. Jensen, and H. Bruus, “A numerical study of microparticle acoustophoresis driven by acoustic radiation forces and streaming-induced drag forces," Lab Chip 12(22), 4617-4627 (2012).

${ }^{17}$ A. D. Pierce, W. L. Siegmann, and E. M. Brown, "Theory of sound propagation through a fluidic medium with suspended clay and silt particles having the general characteristics of mud," J. Acoust. Soc. Am. 145(3), 1787-1787 (2019).

${ }^{18}$ R. I. Jajarmi, "Acoustic separation of submicron particles in gaseous flows," Ph.D. dissertation, Royal Inst. Tech., Stockholm, Sweden, 2015.

${ }^{19}$ A. Passalacqua and R. O. Fox, "Advanced continuum modelling of gasparticle flows beyond the hydrodynamic limit," Appl. Math. Model. 35(4), 1616-1627 (2011).

${ }^{20}$ C. Liu, Z. Wang, and K. Xu, “A unified gas-kinetic scheme for continuum and rarefied flows VI: Dilute dispersed gas-particle multiphase system," J. Comput. Phys. 386(1), 264-295 (2019).

${ }^{21} \mathrm{~K}$. Xu and H. Juancheng, "A unified gas-kinetic scheme for continuum and rarefied flows," J. Comput. Phys. 229(1), 7747-7764 (2010).

${ }^{22}$ J. Huang, K. Xu, and P. Yu, "A unified gas-kinetic scheme for continuum and rarefied flows II: Multi-dimensional cases," Commun. Comput. Phys. 12(3), 662-690 (2012).

${ }^{23} \mathrm{E}$. Cunningham, "On the velocity of steady fall of spherical particles through fluid medium," Proc. R. Soc. A 83(563), 357-365 (1910).

${ }^{24} \mathrm{~K}$. Xu, Direct Modeling for Computational Fluid Dynamics: Construction and Application of Unified Gas-Kinetic Schemes (World Scientific, Singapore, 2015). 
${ }^{25} \mathrm{C}$. Tam, Computational aeroacoustics- $A$ wave number approach (Cambridge, New York, 2012).

${ }^{26} \mathrm{~F}$. Q. Hu, "A perfectly matched layer absorbing boundary condition for linearized Euler equations with a non-uniform mean flow," J. Comput. Phys. 208(2), 469-492 (2005).
${ }^{27}$ C. K. W. Tam and J. C. Webb, "Dispersion-relation-preserving finite difference schemes for computational acoustics," J. Comput. Phys. 107(2), 262-281 (1993).

${ }^{28}$ R. J. Imani and E. Robert, "Estimation of acoustic forces on submicron aerosol particles in a standing wave field," Aerosol Sci. Tech. 52(1), 57-68 (2018). 